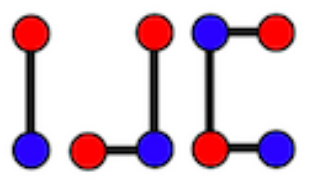

\title{
New proofs of König's bipartite graph characterization theorem
}

\author{
Salman Ghazal ${ }^{\mathrm{a}}$ \\ ${ }^{a}$ Lebanese University, Hadath, Beirut, Lebanon \\ salmanghazal@hotmail.com
}

\begin{abstract}
We introduce four new elementary short proofs of the famous König's theorem which characterizes bipartite graphs by absence of odd cycles. Our proofs are more elementary than earlier proofs because they use neither distances nor walks nor spanning trees.

Keywords: bipartite graph, odd cycle, path

Mathematics Subject Classification : 05C75

DOI: $10.19184 /$ ijc.2017.1.2.4

\section{Introduction}

In this short paper, graphs are finite and may contain loops or multiple edges. The vertex set of a graph $G$ is denoted by $V(G)$ while its edge set is denoted by $E(G)$. The induced subgraph of $G$ by $A \subseteq V(G)$ is denoted by $G[A]$. A subgraph $H$ of $G$ is called a spanning subgraph of $G$ if $V(H)=V(G)$. A set $X$ of pairwise nonadjacent vertices of $G$ is said to be a stable set of $G$, that is $G[X]$ has no edge. With abuse of notation, $x y$ is used to denoted an edge whose endpoints are the vertices $x$ and $y$. The length of a path or a cycle is the number of its edges. A cycle of odd (resp. even) length is called an odd (resp. even) cycle. A path between two vertices $a$ and $b$ is called an $a, b$-path. We do not distinguish between a connected component and the subgraph it induces.
\end{abstract}

Received: 04 Apr 2017, Revised: 12 Aug 2017, Accepted: 20 Aug 2017. 
A graph with no cycle is called acyclic and a connected acyclic graph is called a tree. It is well known that each endpoint of each longest path of an acyclic graph has at most one neighbor. Hence,

Observation 1. Every acyclic graph has a vertex that has at most one neighbor.

A graph $G$ is bipartite if its vertex set is the union of two disjoint (possibly empty) stable sets $X$ and $Y$. In this case, $\{X, Y\}$ is said to be a bipartition of $G$.

We have the following easy observations.

Observation 2. If $\{X, Y\}$ is a bipartition of $G, a \in X$ and $b \in Y$, then $\{X, Y\}$ is again a bipartition of $G+a b$, because $X$ and $Y$ are still stable in $G+a b$.

Observation 3. If $G^{\prime}$ is obtained from $G$ by keeping only one copy of each set of multiple edges of $G$, then $G$ is bipartite if and only if $G^{\prime}$ is so.

Observation 4. A graph $G$ is bipartite if and only if each of its connected components are so.

Proof. Let $A_{1}, \cdots, A_{k}$ be the connected components of a graph $G$. Suppose that $G$ is bipartite and let $\{X, Y\}$ be a bipartition. For any $1 \leq i \leq k$, let $X_{i}=X \cap A_{i}$ and $Y_{i}=Y \cap A_{i}$. Then $\left\{X_{i}, Y_{i}\right\}$ is a bipartition of $A_{i}$. Conversely, suppose that $A_{1}, \cdots, A_{k}$ are bipartite and for each $1 \leq i \leq k$, let $\left\{X_{i}, Y_{i}\right\}$ be a bipartition of the connected component $A_{i}$. Set $X=\cup_{i=1}^{k} X_{i}$ and $Y=\cup_{i=1}^{k} Y_{i}$. Then $\{X, Y\}$ is a bipartition of $G$, hence $G$ is bipartite.

If $\{X, Y\}$ is a bipartition of $G$, then clearly $\{Y, X\}$ is a bipartition of $G$. In general,

Observation 5. Suppose that $\{X, Y\}$ is a bipartition of $G$ and $\left\{X_{i}, Y_{i}\right\}, 1 \leq i \leq k$, form bipartition of its connected components. Then for any $1 \leq i \leq k$, we have $\left\{X^{\prime}, Y^{\prime}\right\}$ form a bipartition of $G$, where $X^{\prime}=\left(X-X_{i}\right) \cup Y_{i}$ and $Y^{\prime}=\left(Y-Y_{i}\right) \cup X_{i}$.

Observation 6. Suppose that $P=x_{1} x_{2} \ldots x_{n}$ is a path in a bipartite graph $G$ with a specified bipartition $\{X, Y\}$. If $a$ and $b$ are adjacent vertices of $G$, then they must be in distinct partite sets. So, if $x_{1} \in X$, then so is every vertex of $P$ with odd index, while every vertex of $P$ with even index is in $Y$.

Hence,

Observation 7. Suppose that $P=x_{1} x_{2} \ldots x_{n}$ is a path in a bipartite graph $G$ with a specified bipartition $\{X, Y\}$. If $x_{1} \in X$, then, $n$ is odd if and only if $x_{n} \in X$.

Therefore,

Observation 8. If $C=x_{1} x_{2} \cdots x_{n} x_{1}$ is a cycle of a bipartite graph $G$, then it must be even, since otherwise the adjacent vertices $x_{n}$ and $x_{1}$ must be in the same partite set, which contradicts its stability.

Thus,

Observation 9. If $G$ is a bipartite graph, then it has no odd cycles. 
In fact, the above obvious necessary condition of bipartite graphs is also sufficient. This is proved in 1936 by König [1]. Proofs of the sufficient condition used distances, walks or spanning trees [2].

Theorem 1.1 (König [1]). A graph is bipartite if and only if it has no odd cycle.

\section{Main Results}

The necessary condition of König's theorem was easy and obvious. Here, we introduce four new elementary proofs of only the sufficient condition of König's theorem that use neither distances nor walks nor spanning trees. We may assume that $G$ has no multiple edges, due to Observation 2 and Observation 3.

\section{First Proof:}

Proof. Let $G$ be a graph that has no odd cycle. Due to Observation 4, we may assume that $G$ is connected. Since $G$ has no loop (because a loop is odd cycle), any vertex of $G$ can be viewed as a bipartite, connected and induced subgraph of $G$. Let $H$ be a maximal bipartite, connected and induced subgraph of $G$. We prove that $G=H$ and consequently we get that $G$ is a bipartite graph. Suppose to the contrary that $G \neq H$. Then $V(H) \neq V(G)$. Since $G$ is connected, $\exists z \in V(G) \backslash V(H)$ and $\exists t \in V(H)$ such that $z t \in E(G)$. Let $\left\{X_{1}, X_{2}\right\}$ be a bipartition of $H$. For $i=1,2$, if $\forall x \in X_{i}, z x \notin E(G)$, then $X_{i} \cup\{z\}$ would be a stable set and thus $G[V(H) \cup\{z\}]$ would be a bipartite, connected, induced subgraph of $G$ and that contains $H$ strictly, which contradicts the maximality of $H$. Hence, for $i=1,2, \exists x_{i} \in X_{i}$ such that $z x_{i} \in E(G)$. However, since $H$ is connected, it contains an $x_{1}, x_{2}$-path $P$. Since $H$ is bipartite and $x_{1}$ and $x_{2}$ are in distinct partite sets, then the length of $P$ is odd (by Observation 7). Therefore, adding to $P$ the edges $z x_{1}$ and $z x_{2}$ forms an odd cycle, which is a contradiction.

\section{Second Proof:}

Proof. Let $G$ be a graph that has no odd cycle. The spanning subgraph of $G$ with no edges is bipartite. Let $H$ be a maximal bipartite spanning subgraph of $G$. We prove that $G=H$ and consequently we get that $G$ is a bipartite graph. Suppose to the contrary that $G \neq H$. Then $E(H) \neq E(G)$ and hence $\exists e=a b \in E(G)-E(H)$. Let $\{X, Y\}$ be a bipartition of $H$. By maximality of $H$, the graph $H^{\prime}=H+e$ is not bipartite and thus $a$ and $b$ lie in the same partite set of $H$, say $X$, since otherwise, $\{X, Y\}$ would be a bipartition of $H^{\prime}$ also (by Observation 2). If there is an $a b$-path $P$ in $H$, then its length is even and adding to it the edge $e$ would create an odd cycle in $G$, a contradiction. Therefore, $a$ and $b$ are in distinct components of $H$. Let $A$ be the component of $H$ containing $a$. Then $X^{\prime}=(X-(X \cap A)) \cup(Y \cap A)$ and $Y^{\prime}=(Y-(Y \cap A)) \cup(X \cap A)$ form a bipartition of $H$ (Observation 5) and thus $H^{\prime}$ (Observation 2, because now $a$ and $b$ are in distinct partite sets of $H$ ). This contradicts the fact that $H^{\prime}$ is not bipartite.

\section{Third Proof:}


Proof. Suppose that there exists a graph that has no odd cycle but it is not bipartite. Let $G$ be a minimal such a counterexample. Let $e=a b \in E(G)$. Then $G-e \subsetneq G$ and $G-e$ has no odd cycle. Hence, $G-e$ is bipartite, by minimality of $G$. Let $\{X, Y\}$ be a bipartition of $G-e$. If $a$ and $b$ are in distinct partite sets, then $\{X, Y\}$ is a bipartition of $G$ as well (Observation 2), a contradiction. So, $a$ and $b$ belong to the same partite set, say $X$. Suppose that there is an $a, b$-path $P \subseteq G$ distinct from $a b$. Then $P \subseteq G-e$. Since $a$ and $b$ are in the same partite set of $G-e$, then the length of $P$ is even (Observation 7). Adding to $P$ the edge $a b$ creates an odd cycle in $G$, a contradiction. So $a b$ is the unique $a, b$-path. Thus $G-e$ is not connected. Let $A$ be the component of $G-e$ containing $a$. Then $b \notin A$. Now $X^{\prime}=(X-(X \cap A)) \cup(Y \cap A)$ and $Y^{\prime}=(Y-(Y \cap A)) \cup(X \cap A)$ form a bipartition of $G-e$ (Observation 5) and thus $G$ (Observation 2), since $b \in X^{\prime}$ and $a \in Y^{\prime}$. A contradiction.

\section{Fourth Proof:}

Proof. First we prove by induction on the number of vertices that if a graph is acyclic, then it is bipartite. Let $F$ be such a graph. Then $F$ has a vertex $x$ that has at most one neighbor $y$ (Observation 1). Since $F-x$ is acyclic as well, then by the induction hypothesis, it is bipartite, with bipartition say $\{A, B\}$. We may assume that $y \notin A$ (if $y$ exist). Then $\{A \cup\{x\}, B\}$ is a bipartition of $F$.

Let $G$ be a graph that has no odd cycle. If $G$ has no (even) cycle, then it is acyclic and thus it is bipartite. Otherwise let $e \in E(C)$, for some even cycle $C$ of $G$. By induction on the number of cycles of $G$, the graph $G-e$ is bipartite since it has fewer cycles than $G$. But the path $P=C-e \subseteq G-e$ has an odd length, hence its endpoints are in distinct partite sets. Thus $G=(G-e)+e$ is bipartite (Observation 2).

\section{References}

[1] D. König, Theorie der endlichen und unenlichen Graphen, Akademische Verlagsgesellschaft (1936).

[2] R. Diestel, Graph Theory, Graduate Texts in Mathematics (2005). Springer. ISBN 978-3-64214278-9 